\title{
Fatigue of Intermittently Stimulated Paralyzed Human Quadriceps During Imposed Cyclical Lower Leg Movements
}

\author{
Henry M. Franken, Peter H. Veltink, Marc Fidder, and Herman B. K. Boom \\ Department of Electrical Engineering, University of Twente, The Netherlands
}

\begin{abstract}
Summary: In this study the torque output of intermittently stimulated paralyzed human knee extensor muscles during imposed isokinetic cyclical lower leg movements was investigated in four paraplegic subjects. During prolonged (10 min) experiments the influence of knee angular velocity and stimulation parameters on fatigue-induced torque decline was studied. Pulse width and amplitude were set to obtain maximal recruitment. The cycle time was maintained constant at $2 \mathrm{~s}$, comparable to a walking cycle. The maximum torque and averaged torque per cycle were estimated to determine the muscle's performance during sustained intermittent stimulation. The overall loss in time of these parameters had a typical exponential decay reaching asymptotic values. Additionally, larger knee velocities resulted in a significantly faster and relatively larger decay of maximum and averaged torque. Also, the rate and relative decrement of torque output during concentric contractions increased with increasing number of pulses in a cycle. Identification trials, determining the (isometric) torque-angle and (isokinetic) torque-angular velocity relation, were performed. The relations appeared to change due to fatigue. The results might be valuable in the design of optimal control systerins for functional electrical stimulation which pursue minimization of muscle fatigue. They may contribute to the derivation of a cost criterion, describing muscle fatig!le as a function of both joint movement and stimulation parameters. Key Words: Quadriceps muscles-Functional electrical stimulation-Paraplegia-Isokinetics-Muscle fatigue.
\end{abstract}

Artificial muscle activation by electrical stimulation is a promising rehabilitation technique for the restoration and enhancement of locomotor functions in patients suffering from spinal cord injury $(1,11,16)$. A major issue in the control of functional electrical stimulation (FES) of paralyzed muscles is the decay of muscle force resulting from fatigue under sustained continuous or intermittent stimula-

Accepted November 9, 1992.

Address correspondence and reprint requests to Dr. H. M. Franken at Biomedical Engineering Division, Department of Electrical Engineering, University of Twente. P.O. Box 217, 7500 AE Enschede, The Netherlands. tion. This decay limits the time a function can be performed. In order to sustain a FES-induced task, such as walking, as long as possible, fatigue should be minimized. This can be achieved by optimization of all parameters that contribute to it. The control law, determining the stimulation parameters, should be based on a cost criterion, describing the dependence of muscle fatigue on joint movement and stimulation parameters. Such a relation has been insufficiently established.

Training can markedly improve the fatigue resistance of paralyzed muscle $(15,19)$. Also, stimulation parameters influence fatigue $(3,6, \mathrm{i} 2)$. Levy et al. 
(12) found that the decay of isometric quadriceps torque during prolonged continuous transcutaneous stimulation, in paraplegics, is muscle length (or knee angle)-dependent and is exponential in time. The knee angle influenced the initial torque according to the length-tension relation of the quadriceps. The exponential decay had a steady-state asymptote of approximately $20 \%$ of the initial torque. This trend is also characteristic for isometric quadriceps torque during submaximal continuous and intermittent transcutaneous stimulation (3). During continuous stimulation, the rate and relative torque decline were independent of the recruitment level, whereas intermittent stimulation exhibited significantly lower rate and relative torque decline compared with continuous stimulation (3). In addition, Carroll et al. (6) showed that, using continuous percutaneous stimulation of isometric quadriceps muscle, higher stimulation frequencies resulted in faster decay of the force output. The relative asymptote also decreased with increasing frequency.

No information was found describing the influence of knee joint movement on quadriceps muscle fatigue during prolonged intermittent stimulation. Fatigue due to dynamic muscle contraction has not been well studied.

The objective of the present study was to contribute to the understanding of the relation between the occurrence of fatigue, the joint movement, and stimulation characteristics. Fatigue was studied at a functional level and was therefore defined as the decrease of output (maximum torque (MT) or averaged torque (AT)) under constant stimulation and movement conditions. A protocol was designed to compare the overall loss of tetanic torque at the knee joint during sustained intermittent stimulation at different isokinetic cyclical knee joint movements. The influence of stimulation parameters was also investigated. The quadriceps-lower leg system was chosen as a convenient example to study fatigue. The implications of the results of this study with respect to the design of optimal control FES systems for paralyzed lower extremity muscles are discussed.

\section{METHODS}

\section{Subjects}

The four subjects who participated in this study were complete T5-T6 level spinal cord injured patients (Table 1). All had normally excitable quadri-
TABLE 1. Specific data about the four paraplegic subjects who participated in this study ${ }^{a}$

\begin{tabular}{llccc}
\hline Subject & Sex & $\begin{array}{c}\text { Year } \\
\text { of birth }\end{array}$ & $\begin{array}{c}\text { Year } \\
\text { of injury }\end{array}$ & $\begin{array}{c}\text { FES } \\
\text { training }\end{array}$ \\
\hline SS & Male & 1966 & 1980 & 6 months \\
JM & Female & 1961 & 1981 & 3 years, \\
& & & 10 months \\
MD & Male & 1964 & 1983 & 6 months \\
TN & Male & 1974 & 1989 & 5 months \\
\hline
\end{tabular}

${ }^{a}$ All subjects were complete T5-T6 level spinal cord-injured patients. Their quadriceps were normally excitable without significant occurrence of spasm.

ceps muscles (without significant occurrence of spasm) and had been enrolled in the FES training program of the Roessingh Rehabilitation Center (Enschede, The Netherlands) for the restoration of locomotor functions for a minimum of 5 months. The program concentrated on lower limb weight training (once a day, $30 \mathrm{~min}, 5-\mathrm{kg}$ ankle load) and standing with a reciprocating gait orthosis (RGO) and crutches (once a week, $30 \mathrm{~min}$, not all patients trained standing regularly) both with quadriceps stimulation (15).

\section{Experimental Set-up}

\section{Dynamometer}

The subjects were seated on a KINCOM 125ES (Kinetic Communicator Exercise System) dynamometer bench (Chattecx Cooperation, Chattanooga Group, Chattanooga, TN, U.S.A.) with the lower leg securely attached to the rotating lever arm just above ankle, as shown in Fig. 1. An electrical motor moved this arm cyclically at various speeds (Table 2). The knee angle range was centered around a knee angle of $50^{\circ}$, at which a maximum in the torque-angle relation was found (12). A constant cycle time was maintained by increasing the angle range at higher velocities (Table 2). The hip angle was fixed at $100^{\circ}$ using straps. A force sensor, composed of a bridge of strain gauges on the lever arm and a built-in bridge amplifier, measured the torque just above the ankle tangentially to the motor axis (8). Knee angle and angular velocity were measured at the motor axis. Testing with known weights at static angles proved correct tangential measurement and accuracy and linearity of the force sensor (largest force error $<3 \%$ applied load). Angle measurements were verified with a leveling instrument and external goniometer to measure rel- 


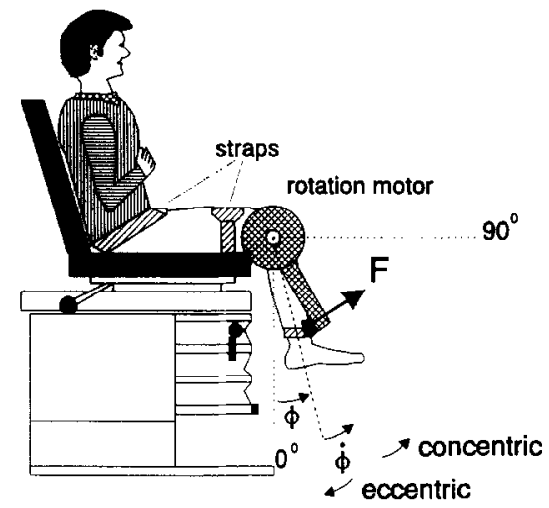

FIG. 1. Schematic of experimental set-up. The angular position and velocity, measured at the motor axis, are defined positively during extension with zero as indicated. The patient is strapped at the hip and above the knee to measure knee torque only and to ensure static position of the body. A force sensor, composed of a bridge of strain gauges on the lever arm and a built-in bridge amplifier, measured the knee torque just above the ankle tangentially to the motor axis.

ative angles (largest angle error $<0.1^{\circ}$ ). Velocity measurements were confirmed successfully using numerical differentiation of the angle signal. The largest speed error appeared to be smaller than $1 \% \mathrm{~s}$ in the range where stimulation was applied. The accuracy of the sensors agree with the results of an extensive examination of the KINCOM dynamometer by Farrell and Richards (8). The effect of these small errors is negligible in comparison with the possible errors made in estimating the physiological knee angle.

\section{Stimulation and Data Recording}

The quadriceps of the strongest leg was stimulated using two $5 \times 9-\mathrm{cm}$ adhesive surface electrodes (Pals, Axelgaard Manufacturing Co., Fallbrook, CA, U.S.A.). The cathode and anode were placed over the motor points of rectus femoris/ vastus lateralis and vastus medialis, respectively. This placement of electrodes for quadriceps conformed to clinical usage during paraplegic standing (15). The stimulation was controlled by an IBM-ATcompatible computer with AD facilities (Analog Devices, RTI-815, 12-bit) and a current stimulator, generating monophasic rectangular pulses. Pulse width and pulse amplitude were set to obtain maximal recruitment. In the control of FES-induced dynamic muscle contraction, complications arising by the nonlinear relationship between these stimulation parameters and torque (i.e., recruitment curve), can often be avoided using maximal recruit- ment $(1,23)$. The interpulse intervals were 20 and 40 $\mathrm{ms}$, both ensuring a fused contraction. Force, angular position, and velocity were sampled at 100 Hz. These samples and the stimulus data were stored on disk for off-line analysis.

\section{Stimulation Parameters}

The following nomenclature of stimulation parameters will be used in this study. The cycle time is the period of repetition. The on-time is the burstlength of a sequence of stimulation pulses during one cycle. The duty cycle is the on-time divided by the cycle time. During continuous stimulation the duty cycle is $100 \%$. Interpulse interval (IPI) is the time between the onset of subsequent pulses and thus, if constant, is equal to one divided by the stimulation frequency. The number of pulses given in one cycle is equal to the on-time divided by the IPI, assuming IPI is constant. Thus, the number of pulses depends on duty cycle and IPI. The amplitude of the stimulation pulses is the pulse amplitude. The pulse width is the duration of one stimulation pulse.

\section{Protocol}

The measurement system for force, angle, and velocity was calibrated at the beginning of the experiment.

Quadriceps muscle behavior of each patient was identified in short sessions at the beginning, middle,

TABLE 2. Parameter settings during subsequent trials ${ }^{a}$

\begin{tabular}{ccccccc}
\hline & $\begin{array}{c}\text { Angle } \\
\text { Trial } \\
(\mathrm{deg} / \mathrm{s})\end{array}$ & $\begin{array}{c}\text { range } \\
(\mathrm{deg})\end{array}$ & Trigger & $\begin{array}{c}\text { Duty } \\
\text { cycle } \\
(\%)\end{array}$ & $\begin{array}{c}\text { IPI } \\
(\mathrm{ms})\end{array}$ & $\begin{array}{c}\text { Number } \\
\text { of } \\
\text { pulses }\end{array}$ \\
\hline 1 & 0 & 50 & 2 s timing & 32 & 20 & 32 \\
2 & 20 & $41.5-59$ & $42^{\circ}$ & 32 & 20 & 32 \\
3 & 40 & $32-68$ & $35^{\circ}$ & 32 & 20 & 32 \\
4 & 40 & $32-68$ & $35^{\circ}$ & 32 & 40 & 16 \\
5 & 40 & $32-68$ & $42^{\circ}$ & 16 & 20 & 16 \\
6 & 80 & $6.5-80.5$ & $11^{\circ}$ & 32 & 20 & 32 \\
7 & -40 & $32-68$ & $65^{\circ}$ & 32 & 20 & 32 \\
\hline
\end{tabular}

a Amplitude of stimulation and pulse width were determined before the experiment on the basis of a recruitment curve (maximal recruitment) and kept constant during the entire session. The cycle time was $2 \mathrm{~s}$. The angle movement was a cyclical triangular pattern. Positive velocities correspond to concentric contractions, negative velocities to eccentric contractions. The influence of joint velocity on fatigue was determined from trials $1-3,6$, and 7 . The influence of duty cycle, IPI, and the number of pulses per cycle was determined in a concentric contraction at $40 \% \mathrm{~s}$ in trials $3-5$. 
and end of the whole experiment. The active torque-angle relation was measured at various static knee angles. The active torque-angular velocity relation was measured isokinetically at a knee angle of $50^{\circ}$ (Fig. 1). Each muscle response was elicited by a stimulation burst of $640 \mathrm{~ms}$. The relations obtained at the beginning of the experiment correspond to unfatigued muscle status.

Each patient participated in the seven fatigue trials indicated in Table 2 during one day. Each trial lasted $10 \mathrm{~min}$ followed by $45 \mathrm{~min}$ of rest, which was assumed to be adequate for recovery between subsequent trials $(3,15,18)$. During the rest period, the patient was moderately physically active, while sitting in a wheelchair. Stimulation electrodes remained on the sites during the entire session.

Table 2 also shows the order in which the fatigue trials were performed, with the following remarks: (a) A warm-up trial was performed in order to make the first trial comparable to other trials. It consisted of $5 \mathrm{~min}$ of intermittent isometric stimulation before the beginning of the entire protocol, followed by 20 min of rest. The pulse amplitude was chosen to obtain $80 \%$ recruitment (3). The remaining parameters were identical to trial 1 . (b) The onset of the stimulation was triggered so that the muscular activity occurred around the optimal knee angle $\left(50^{\circ}\right)$ with constant velocity and during one phase of the swing. The stimulation was time-triggered in the isometric trials. (c) Velocities higher than $80 \%$ sere avoided because the angle range was limited to $80^{\circ}$ (Fig. 1) and the cycle time was set to $2 \mathrm{~s}$. (d) The eccentric trial, no. 7, was performed last because it was feared that it would cause spasm during subsequent trials. (e) The active torque generated by the stimulated quadriceps was estimated by subtracting the passive torque from the measured total torque. The passive torque was estimated at the beginning of each trial by measuring the torque due to gravitation and elasticity in the joint, i.e., without stimulating the quadriceps, while the lower leg was moving according to the movement parameters of the trial. Ten cycles were measured and subsequently averaged.

Before the commencement of each trial the muscle was warmed up by passive joint movements at $80 \%$ for approximately $1 \mathrm{~min}$ and 10 isometric contractions (knee angle $=50^{\circ}$, pulse width $=300 \mu \mathrm{s}$, pulse amplitude $=100 \mathrm{~mA}$, IPI $=20 \mathrm{~ms}$, duty cycle $=50 \%$, cycle time $=2 \mathrm{~s}$ ). The required pulse amplitude for maximal recruitment was determined with an isometric recruitment curve (knee angle: $50^{\circ}$, pulse width 300 or $500 \mu$ s, pulse amplitude: 0 to $100 \mathrm{~mA} ; 10 \mathrm{~s}$ up, $10 \mathrm{~s}$ down). This curve also served to test the muscle recovery following the rest period after each trial (7). Each patient achieved sufficient recovery from fatigue, since the torques generated at maximal recruitment at the beginning of a trial were comparable (deviation from mean $<10 \%$ ). The pulse amplitude and pulse width appeared to be constant during the entire experiment, since maximal recruitment was achieved in each recruitment trial with the same pulse width and amplitude.

\section{Off-Line Data Analysis}

The active torque yielded the maximum torque (MT) and the averaged torque (AT) per swing by calculation. MT was determined after filtering the active torque with a 100 -ms moving average window. AT was determined by dividing the torquetime integral by the duration of half a cycle (the phase of the siving in which the elicited muscle activity takes place). Fatigue parameters, describing the decay of the MT and AT per cycle as a function of time (one sample per cycle with each cycle being $2 \mathrm{~s})$, were derived by curve-fitting the following equation to the data:

$$
\xi(t)=\xi_{\max } \cdot\left[\left(1-\xi_{0}\right) \cdot \exp \left(-t / \tau_{\xi}\right)+\xi_{0}\right]
$$

where $\xi$ is either MT or AT, $t$ is time, $\xi_{\max }$ is the maximum achieved in the considered trial, $\tau_{\xi}$ is the time constant of decay in $\xi$ due to fatigue, and $\xi_{\mathrm{o}}$ is the relative asymptotic value to which $\xi(t)$ descends. These parameters are referred to as "fatigue parameters" in the rest of this paper. The first part of the data (approximately 10 cycles), in which $\xi$ increases to $\xi_{\max }$, was ignored. Equation (1) was fitted iteratively by minimizing the resulting root mean square (RMS) deviation from the experimental data (see also (3)).

The RMS error of fitting Eq. (1) to the data was small (RMS error $<2 \%$ for trials $1-3,6$, and 7 ). A larger RMS error was found for trials with a smaller number of pulses per cycle (RMS error $<10 \%$ for trials 4 and 5).

\section{RESULTS}

Figure 2 shows typical results of the torque output at the knee joint, knee angle, and angular velocity during one swing with intermittent stimulation of the quadriceps in the forward swing resulting in a concentric contraction. From the active torque, 


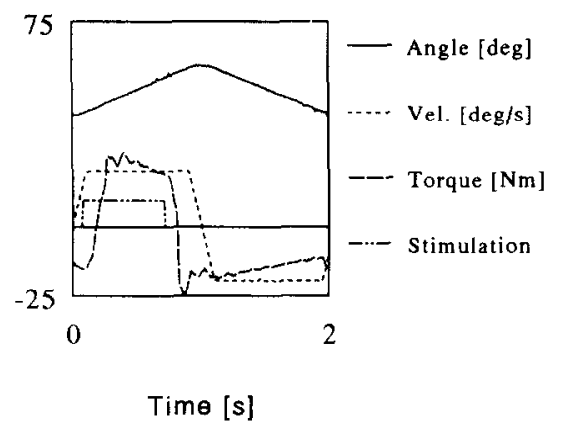

FIG. 2. Total knee torque, angular position, velocity, and the applied stimulation burst, for one cycle with concentric contraction. The angular velocity is constant during the muscle contraction.

estimated by subtracting the averaged passive torque from the measured total torque at the knee, MT and AT per swing were calculated (Fig. 3).

\section{Muscle Behavior}

Figure 4 depicts torque-angle and torque-angular velocity relations, obtained in the muscle behavior identification experiments in subject TN. The shape of the depicted relations in Fig. 4 is representative for all patients. The maximum values are different for all patients. The torque-angle relation exhibits a maximum at the optimal knee angle. The torqueangular velocity relation conforms to Hill's equation (10). The torque decrement with increasing angular velocity is consistent with a hyperbolic relation. Noteworthy, the first muscle behavior identification trial was performed before the first fatigue trial, whereas the second and third muscle behavior identification trials were performed directly following fatigue trials 3 and 7 , respectively. The tendency of the output does not alter significantly due to fatigue induced by the preceding trial. The position of the curves is typical for all patients in that the identification at the end of the day resulted in the lowest output.

\section{Fatigue Trials}

Figure 5 illustrates the typical results of MT decay at different isokinetic velocities of patient SS. The exponential decay to steady-state values is characteristic for the decay in MT and AT for all tests with all subjects.

\section{Influence of Knee Angular Velocity}

The influence of knee angular velocity was determined in trials $1-3,6$, and 7 . In these trials the stim- ulation parameters were unchanged whereas the knee angular velocity and consequently the knee angle range were varied, as shown in Table 2.

Figures $6 \mathrm{a}$ and $\mathrm{b}$ display the maximum of MT and AT per cycle $\left(\mathrm{MT}_{\max }\right.$ and $\mathrm{AT}_{\text {max }}$ ) achieved in trials $1-3,6$, and 7 . Each trial has a different knee angular velocity (Table 2). The curves resemble the torqueangular velocity relation in Fig. 4 (first muscle behavior identification trial), indicating comparable condition of the quadriceps muscle before the trials (sufficient recovery). Clearly, the magnitude of torque generated by the stimulated quadriceps under identical conditions significantly differs for all patients (not tested statistically). This is of interest in comparing other fatigue parameters such as time constant and relative asymptotic value.

Figures $6 \mathrm{c}$ and $\mathrm{d}$ present the time constants $\left(\tau_{\mathrm{MT}}\right.$ and $\tau_{\text {AT }}$ ) obtained by fitting Eq. (1) to the data. They also appear to depend nonlinearly on the knee angular velocity. This was found for all patients. The correlation of the time constants with knee joint velocity of the pooled data of all patients is significantly negative ( $\alpha \leqslant 0.01$; Table 3 ). The time constant of fatigue thus relates inversely to knee joint velocity.

Figures $6 \mathrm{e}$ and $\mathrm{f}$ display the relative asymptotes to which MT and AT per cycle descend MT $_{\mathrm{o}}$ and $\mathrm{AT}_{\mathrm{o}}$ ) during the trials. The correlation of the relative asymptote with velocity of the pooled data of all patients is again negative but not significant $(\alpha \leqslant$ 0.1 ; Table 3). The dependency of time constant and the relative asymptote of the decay in MT and AT on knee angular velocity is similar. Figures $7 a$ and $b$ depict the significant positive correlation $(\alpha \leqslant 0.01$;

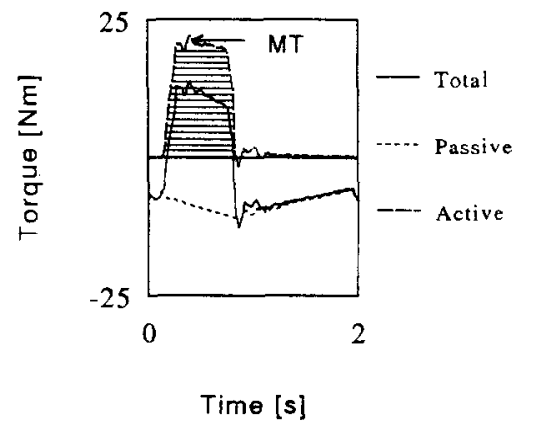

FIG. 3. The torque generated by the stimulated quadriceps was estimated by subtracting the averaged passive torque, measured during 10 passive swings before a trial, from the measured torque during a trial. The maximum torque was determined after filtering the active torque with a $100-\mathrm{ms}$ moving average window. The averaged torque was obtained by dividing the integrated active torque (shaded area) by the duration of half a cycle in which the contraction takes place. 


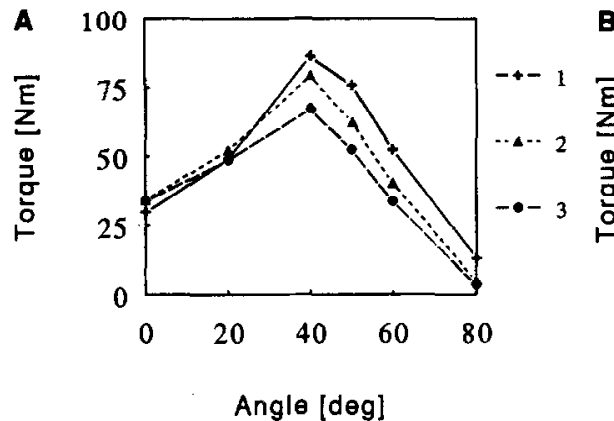

Angle [deg]

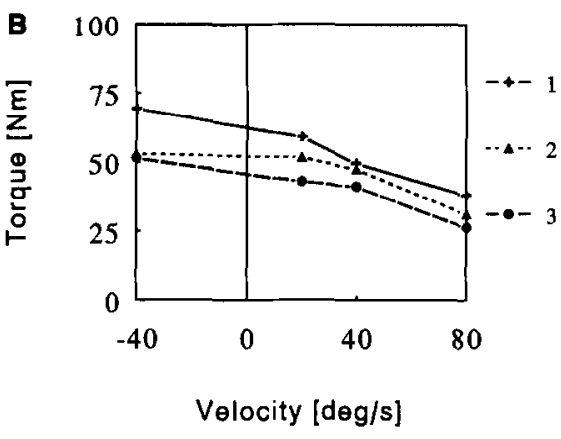

Velocity [deg/s]
FIG. 4. Quadriceps muscle behavior identification results of patient TN. 1: Prior to fatigue trial 1.2: After fatigue trial 3. 3: After fatigue trial 7. Stimulation parameters: $\mid \mathrm{PI}=20 \mathrm{~ms}$, pulse amplitude $=100 \mathrm{~mA}$, and on-time $=$ $640 \mathrm{~ms}$. a: Typical registration of the active torque-angle relation of the stimulated quadriceps muscle. The stimulation was applied in isometric conditions. The displayed torque is the averaged active torque over a 100$\mathrm{ms}$ window at the end of the burst. $b$ : Active torque-angular velocity relation. Stimulation was applied during isokinetic knee extension, identical to a fatigue trial. The displayed active torque is the average over a $100-\mathrm{ms}$ window measured around a knee angle of $50^{\circ}$.
Table 3) of the time constant with the relative asymptote of the decay of the pooled data of all patients in MT and AT respectively.

\section{Constant IPI, Varying Duty Cycle and Number of Pulses}

The influence of duty cycle (and consequently number of pulses) at constant IPI and knee joint velocity was determined in trials 3 and 5 (Fig. 8: trials 3 and 5). The on-time chosen was $640 \mathrm{~ms}$ in trial 3 and $320 \mathrm{~ms}$ in trial 5, resulting in a duty cycle of 32 and $16 \%$, respectively. Consequently, the number of pulses is 32 in trial 3 and 16 in trial 5 (Table 2).

Figures $8 \mathrm{a}$ and $\mathrm{b}$ display $\mathrm{MT}_{\max }$ and $\mathrm{AT}_{\max }$ for the two duty cycles. Clearly, the magnitudes of both $\mathrm{MT}_{\max }$ and $\mathrm{AT}_{\max }$ do not double with twice the duty cycle. This could be expected for $\mathrm{MT}_{\max }$ because maximal torque will be obtained within 320 ms. Noteworthy is the slight increase in $\mathrm{MT}_{\max }$ in subjects SS, JM, and MD for trial 5 in comparison with trial 3, whereas subject TN shows a slight decrease. The relative difference between $\mathrm{AT}_{\max }$ obtained in the two trials can be explained by the knee angle range in which contraction takes place. Because in both trials the contraction is centered around the optimal angle in the torque-angle relation, $\mathrm{AT}_{\max }$ obtained with a duty cycle of $16 \%$ will be larger than half of $\mathrm{AT}_{\max }$ obtained with a duty cycle of $32 \%$.

$\tau_{M T}$ and $\tau_{\text {AT }}$ inversely relate to the duty cycle (Figs. 8c and d). This was true for all patients except SS, in which the decay in MT is faster for a smaller duty cycle.

$\mathrm{MT}_{\mathrm{o}}$ and $\mathrm{AT}_{\mathrm{o}}$ for the different duty cycles is depicted in Figs. $8 \mathrm{e}$ and $\mathrm{f}$. The relative asymptote for a duty cycle of $16 \%$ is higher than for a duty cycle of $32 \%$ for each patient. Thus, relative magnitude of fatigued steady-state to the unfatigued condition (for concentric contractions) inversely relates to duty cycle.

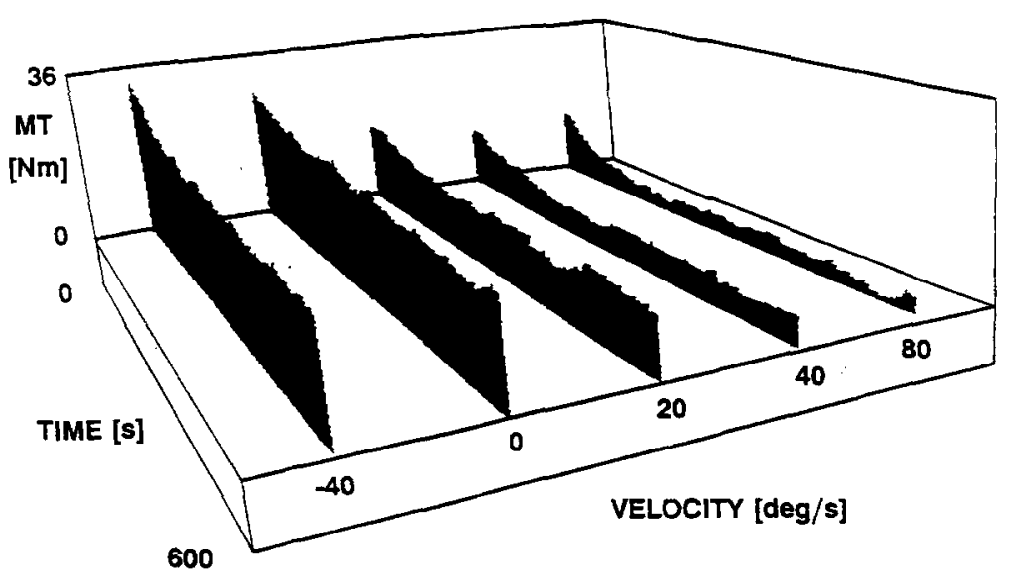

FIG. 5. Decay of maximum torque per cycle during several fatigue trials with patient SS. The time axis refers to 300 consecutive cycles. The different velocities refer to separate fatigue trials with identical stimulation parameters (Table 2). The maximum torque per cycle is determined as depicted in Fig. 3 . The curve of unfatigued maximum torque as a function of angular velocity (found by connecting the start values of the trials) resembles the torque-angular velocity relation depicted in Fig. 4. 

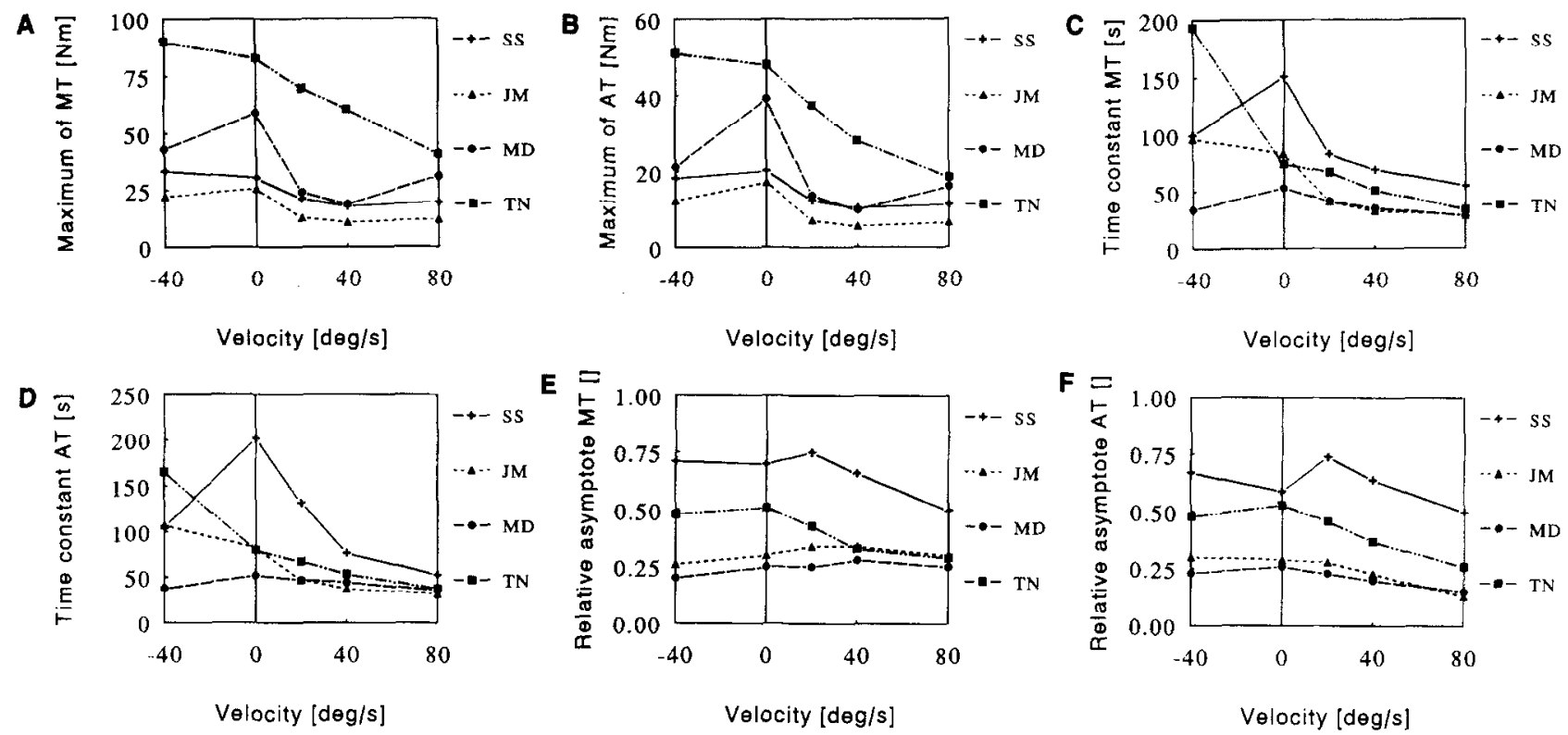

FIG. 6. Varying knee joint velocity: fatigue parameters obtained in trials $1-3,6$, and 7 . The different velocities (measurement points) refer to separate fatigue trials with identical stimulation parameters (Table 2). The patients SS, JM, MD, and TN are indicated in the graph. a: Maximum of maximum torque per cycle (MT max $)$. b: Maximum of averaged torque per cycle (AT max). c: Time constant for decay in maximum torque per cycle $\left(\tau_{M T}\right)$. d: Time constant for decay in averaged torque per cycle $\left(\tau_{A T}\right)$. e: Relative asymptote for decay in maximum torque per cycle $\left(\mathrm{MT}_{\mathrm{o}}\right)$. f: Relative asymptote for decay in averaged torque per cycle $(\mathrm{AT}$ o).

\section{Constant Duty Cycle, Varying IPI and Number of Pulses}

The influence of IPI (and consequently number of pulses) at constant duty cycle and knee joint velocity was investigated in trials 3 and 4 (Fig. 8, trials 3 and 4). The IPI was set to $20 \mathrm{~ms}$ in trial 3 and $40 \mathrm{~ms}$ in trial 4 , resulting in 32 and 16 pulses per cycle, respectively (Table 2 ).

Clearly, $\mathrm{MT}_{\max }$ and $\mathrm{AT}_{\max }$ increase with decreasing IPI (Figs. 8a and b). This has also been reported for isometric contractions (6).

The rate of fatigue has been shown to be faster for shorter IPI during continuous stimulation (6). The rate of fatigue was also investigated for intermittent stimulation in this study. Patients JM and TN satisfied this expectation, SS and MD did not (see Figs. $8 \mathrm{c}$ and d).

TABLE 3. Correlation coefficients for the relations $\tau_{\xi}$ vs. velocity, $\xi_{o}$ vs. velocity, and $\xi_{o}$ vs. $\tau_{\xi}$, where $\xi$ is either $M T$ or $A T^{a}$

\begin{tabular}{lccc}
\hline$\xi$ & $\begin{array}{c}\tau_{\xi} \text { vs. } \\
\text { velocity }\end{array}$ & $\begin{array}{c}\xi_{\mathrm{o}} \text { vs. } \\
\text { velocity }\end{array}$ & $\xi_{\text {o }}$ vs. $\tau_{\xi}$ \\
\hline MT & -0.61 & -0.15 & 0.55 \\
AT & -0.54 & -0.30 & 0.68 \\
\hline
\end{tabular}

${ }^{a}$ Data of all patients has been pooled. Thus $n-20$ (4 patients, trials $1-3,6$, and 7 ).
The relative value of fatigued steady-state to the unfatigued condition for concentric contractions is in direct relation to IPI. $\mathrm{MT}_{\mathrm{o}}$ and $\mathrm{AT}_{\mathrm{o}}$ with $\mathrm{IPI}=40$ $\mathrm{ms}$ are larger than with IPI $=20 \mathrm{~ms}$ for all patients.

\section{Constant Number of Pulses, Varying IPI and Duty Cycle}

In trials 4 and 5 , the duty cycle is 32 and $16 \%$ and IPI is 40 and $20 \mathrm{~ms}$, respectively (Fig. 8 , trials 4 and 5). Thus, the number of pulses is 16 for these two trials. For this case, the influence of both IPI and duty cycle can be studied simultaneously at constant knee joint velocity. Examining Figs. 8c-f does not provide a conclusion as to any superior influence of either IPI or duty cycle. This could partly be due to the larger RMS error of the fit (RMS error $<10 \%$ ).

The combined results of trials 3-5 indicate that, for a given task, duty cycle and 1/IPI should be minimized to postpone fatigue. Thus, the number of pulses should be minimized.

\section{DISCUSSION}

The overall loss of MT and AT during sustained intermittent stimulation under isokinetic condi- 
A

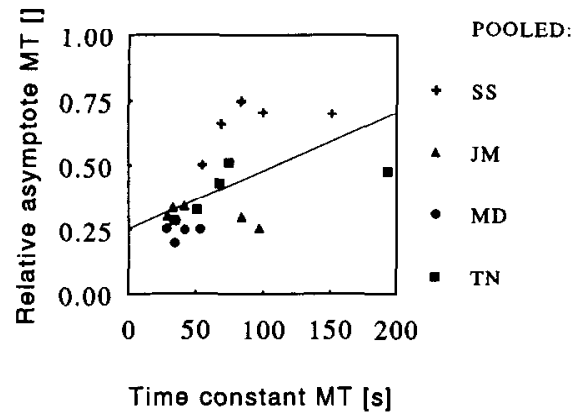

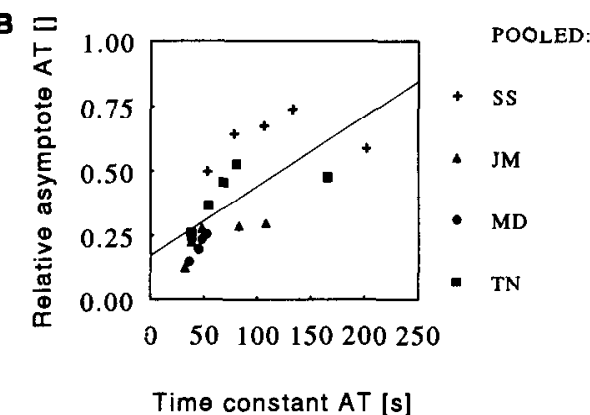

FIG. 7. Relationship between the relative asymptote and the time constant of decay of (a) maximum torque and (b) averaged torque. The drawn line is the linear regression of the pooled data of all patients. The displayed data points correspond to Figs. $6 c-f$. The patients SS, JM, MD, and $T N$ are indicated in the graph. tions, as found in this study, corresponds to the results from fatigue experiments under isometric conditions published by other researchers $(3,4,6$, 12). The typical exponential decay of quadriceps torque, reaching asymptotic values during transcutaneous $(3,12)$, percutaneous $(6)$, and direct (femoral) nerve stimulation (4) is also a well-documented finding. The effect of duty cycle for short cycle times during isometric stimulation was reported by Boom et al. (3). However, to the best of the authors' knowledge, the influence of knee angular velocity and stimulation parameters (duty cycle, IPI, and number of pulses) on the fatigue of intermittent transcutaneously stimulated human quadriceps, during imposed isokinetic cyclical lower leg movements, was never documented.

Electrically stimulated muscle is a nonlinear dy- namic system, exhibiting nonlinear dependence of its produced force on factors such as muscle length and contraction velocity (or joint position and velocity), joint load, fatigue, and temperature. These characteristics complicate the control of the system. The knee angular position dependence was already noted in paraplegic patients $(9,12,20)$. The Hill relation (10) is favored by many researchers for curve-fitting the torque-angular velocity relation of muscles (e.g., 2, 21, 22, 24). Our results conform to this relation. Triolo et al. (20) have already documented the torque-angular velocity relation for intramuscularly stimulated quadriceps muscles in two teen-age spinal cord-injured subjects. To control the system, the dependence of these relations on fatigue for human quadriceps should be known, but have not been documented before. Our results in-
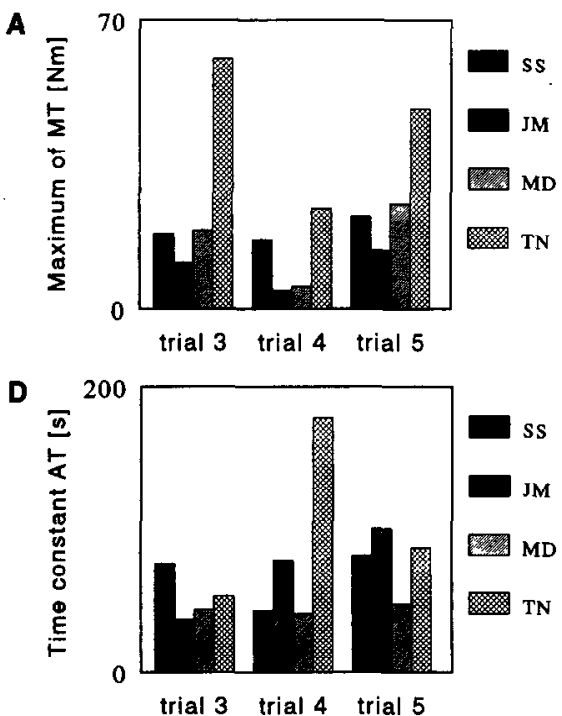

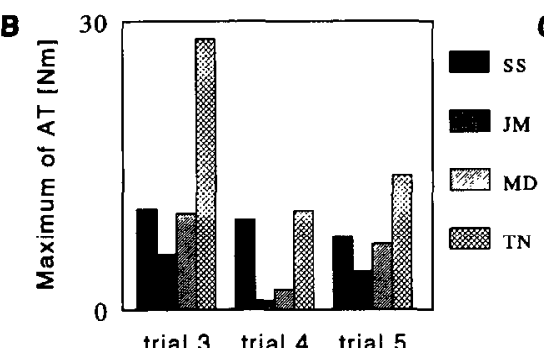

trial $3 \quad$ trial 4 trial 5

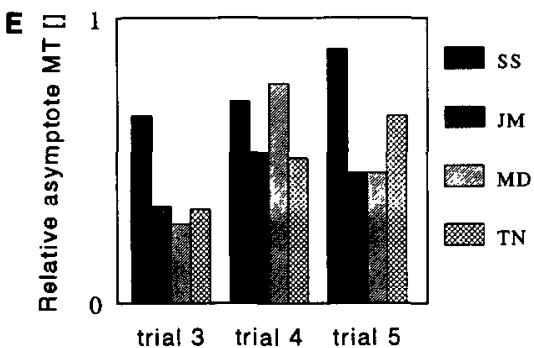

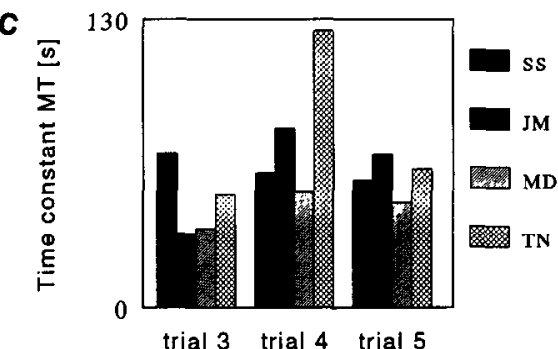

trial 3 trial 4 trial 5

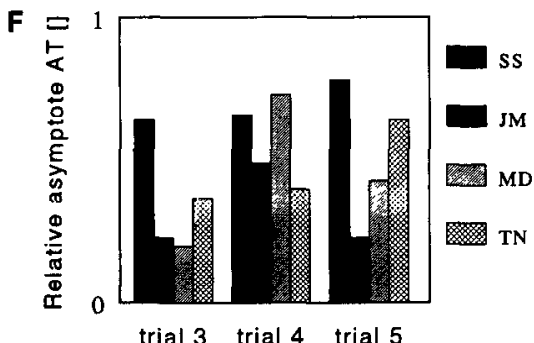

FIG. 8. Varying stimulation parameters: fatigue parameters obtained in trials $3-5$. The knee angular velocity (40\%) and the angle range $\left(32-68^{\circ}\right)$ were kept constant. The duty cycle was $32 \%$ in trial 3 and 4 and $16 \%$ in trial 5 . The IPI was 20 ms in trial 3 and 5 and 40 ms in trial 4 . Thus, the number of pulses was 16 in trial 4 and 5 and 32 in trial 3 . The patients SS, JM, MD, and TN are indicated in the graph. a: Maximum of maximum torque per cycle $\left(\mathrm{MT}_{\max }\right.$ ). b: Maximum of averaged torque per cycle (AT max). c: Time constant for decay in maximum torque per cycle $\left(\tau_{M T}\right)$. d: Time constant for decay in averaged torque per cycle $\left(\tau_{A T}\right)$. e: Relative asymptote for decay in maximum torque per cycle $\left(M T_{0}\right)$. $f$ : Relative asymptote for decay in averaged torque per cycle (ATo). 
dicate that the torque-angular velocity relation alters in shape due to fatigue. It derives from the finding that the relative decrement of torque output due to fatigue is larger during higher knee angular velocity.

The change in the torque-angular velocity relation of animal muscles due to fatigue has been discussed in the literature. There are suggestions that a dramatic metabolic down-regulation by low adenosine triphosphate (ATP) or high inosine monophosphate (IMP) concentrations might be the cause of a relatively higher reduction in torque output (in time) at higher contraction velocities $(5,13)$. In other words, the biochemical process in the muscle may be less efficient at higher contraction velocities. Mizrahi et al. (14) have incorporated several metabolic profiles during fatigue and recovery to model muscle contraction in continuous and intermittent stimulation. This may lead to a better understanding of the metabolic processes during FES-induced muscle contraction. Alternatively, it might be expected that the fast-twitch fibers become completely exhausted, as Salmons and Hendriksson (19) have shown for cat muscles. This leaves a torque-velocity relation which is completely determined by the slow twitch fibers. In principle, this hypothesis could be tested experimentally since it has been known for a long time that the curvature in the torque-velocity relation for fast and slow twitch fibers is significantly different (17). The angular velocity range used in this study, however, was too limited to investigate accurately this curvature change. Another point worth considering is the amount of mechanical work performed (mechanical work $=\int([$ velocity $] *$ [active torque $\left.\left.]\right) d t\right)$. The data indicate that more mechanical work is performed at higher velocities. No mechanical work is performed during isometric contractions, whereas eccentric contractions perform negative mechanical work. Fatigue parameters can, therefore, only be partly correlated to the mechanical work performed.

Precautions were taken to avoid effects of subject movement during and repositioning at the beginning of a trial. Subjects were asked to avoid extensive activity for a few days before the experiment. Small intersubject differences may, however, be explained by the above. Greater intersubject differences may be expected when the period and intensity of training is significantly different (19) (Table 1). Other parameters of the subjects, such as sex, age, and time after injury might also explain intersubject differences (Table 1). It is also noteworthy that the error in the fit of trials 4 and 5 is relatively large (RMS error $<10 \%$ ). This uncertainty must be taken into account when directly interpreting small variations in the fatigue parameters.

It can be argued that the chronological order of the fatigue trials in this study might have been of influence on the achieved results. To investigate this, part of the protocol has been performed in reverse order (trials 6,3 , and 1) on patient JM. The achieved fatigue parameters were reproducible within $10 \%$, indicating that the order was not of significant influence on the results.

\section{CONCLUSIONS}

The relations between stimulation and movement parameters and the occurrence of fatigue can be used in the design of optimal control strategies for FES-induced steady-state cyclical leg movements, such as walking, which pursue minimization of muscle fatigue. Our results may contribute to the derivation of a cost criterion, describing muscle fatigue as a function of both joint movement and stimulation parameters. Summarizing, fatigue can be characterized as a time dependency of functional output of the muscle (e.g., MT, AT) during a fixed stimulation protocol. Based on the presented results, the cost criterion should contain the following relations. In the case of supramaximal stimulation (i.e., pulse amplitude and pulse width set to obtain maximal recruitment), the task should be performed with a minimum number of stimulation pulses per cycle. The pulses should be given in a phase of the movement cycle with the smallest possible angular velocity. As a first approach $\tau$ could be used as a measure of fatigue rate and the relative asymptote as a measure of fatigue magnitude. For optimal control of a cyclical leg movement the stimulation should then be chosen such that $\tau$ and the relative asymptote are maximized (i.e., postponing fatigue as long as possible), while satisfying the boundary conditions imposed by the task. The results, presented in this paper, indicate that $\tau$ and the relative asymptote are highly correlated. Thus, maximizing $\tau$ is sufficient. If $\tau$ is relatively large for a given task, adaptation of the stimulation parameters in time to compensate for fatigue will be small. If the relative asymptote for the given task approaches 1 , fatigue is no limitation on performing this task as long as necessary.

Acknowledgment: We thank the researchers of the Roessingh Rehabilitation Center (Enschede, The Nether- 
lands) and the subjects for their cooperation. This work was supported by the Mobility Restoration (MORES) program of the University of Twente (Enschede, The Netherlands).

\section{REFERENCES}

1. Andrews BJ, Barnett RW, Phillips GF, Kirkwood CA: Rulebased control of a hybrid FES orthosis for assisting paraplegic locomotion. Automedica 11:175-199, 1989.

2. Audu ML, Davy DT: The influence of muscle model complexity in musculoskeletal motion modeling. J Biomed Eng 107:147-157, 1985.

3. Boom HBK, Mulder AJ, Veltink PH: Fatigue during finite state control during neuromuscular stimulation. Prog Brain Res, in press, 1993.

4. Brindley GS, Polkey CE, Rushton DN: Electric splinting of the knee. Paraplegia 16:428-435, 1979.

5. Cady EB, Elshove H, Jones DA, Moll A: The metabolic causes of slow relaxation in fatigued human skeletal muscle. J Physiol 418:327-337, 1989.

6. Carroll SG, Triolo RJ, Chizeck HJ, Kobetic R, Marsolais EB: Tetanic response of electrically stimulated paralyzed muscle at varying interpulse interval. IEEE Trans Biomed Eng BME-36:644-653, 1989.

7. Durfee WK, Maclean KE: Methods for estimating isometric recruitment curves of electrically stimulated muscle. IEEE Trans Biomed Eng BME-36:654-667, 1989.

8. Farrell M, Richards JG: Analysis of the reliability and validity of the kinetic communicator exercise machine. Med Sci Sports Exerc 18:44-49, 1986.

9. Hausdorff JM, Durfee WK: Open-loop position control of the knee joint using electrical stimulation of the quadriceps and hamstrings. Med Biol Eng Comput 29:269-280, 1991.

10. Hill AV: The heat of shortening and the dynamic constants of muscle. Proc R Soc (London) B126:136-195, 1938.

11. Krali A, Bajd T, Turk R, Krajnik J, Benko H: Gait restoration in paraplegic patients: a feasibility demonstration using multichannel surface electrode FES. I Rehab Res Dev 20: 3-20, 1983.

12. Levy M, Mizrahi J, Susak Z: Recruitment, force and fatigue characteristics of quadriceps muscles of paraplegics isometrically activated by surface functional electrical stimulation. J Biomed Eng 12:150-156, 1990.

13. Lodder MAN, Haan A de, Sargeant AJ: Effect of shortening velocity on work output and energy cost during repeated contractions of the rat EDL muscle. Eur J Appl Physiol 62:430-435, 1991.

14. Mizrahi J, Levy M, Kushnir T, Gait Y: Fatigue and recovery of paralyzed quadriceps muscle activated by FES. In: Proceedings 1st International FES Symposium, Sendai, Japan, July 23-25, pp 15-22, 1992.

15. Mulder AJ, Boom HBK, Hermens HJ, Zilvold G: Artificialreflex stimulation for FES-induced standing with minimum quadriceps force. Med Biol Eng Comput 28:483-488, 1990.

16. Peckham PH: Functional electrical stimulation: current status and future prospects of applications to the neuromuscular system in spinal cord injury. Paraplegia 25:279-288, 1989.

17. Petrofsky JS, Phillips CA: The influence of recruitment order and fibre composition on the force-velocity relation and fatiguability of skeletal muscles in the cat. Med Biol Eng Comput 18:381-390, 1980 .

18. Pournezam M, Andrews BJ, Baxendale RH, Phillips GF, Paul JP: Reduction of muscle fatigue in man by cyclical stimulation. J Biomed Eng 10:196-200, 1988.

19. Salmons $S$, Hendriksson J: The adaptive response of skeletal muscle to increased use. Muscle Nerve 4:94-105, 1981

20. Triolo RJ, Robinson DE, Betz RR: Force-velocity and length-tension properties of stimulated human quadriceps in spinal cord injured children. In: Proceedings IEEE EMBS 11th International Congress, pp. 967-968, 1989.

21. Winters JM, Stark L: Analysis of fundamental human movement patterns through the use of in-depth antagonist muscle models. IEEE Trans Biomed Eng BME-23:826-839, 1985.

22. Wilkie DR: Relation between force and velocity in human muscle. J Physiol (London) 110:249-280, 1950.

23. Veltink PH: Control of FES-induced cyclical leg movements of the lower leg. Med Biol Eng Comput 29:NS8-NS12, 1991.

24. Zajac FE: Muscle and tendon: Properties, models, scaling, and application to biomechanics and motor control. Crit Rev Biomed Eng 17:359-410, 1989. 